\title{
On the thermo-poro-mechanics of chemically active faults
}

\author{
Manolis Veveakis \\ Civil and Environmental Engineering, Duke University \\ manolis.veveakis@duke.edu
}

\begin{abstract}
Shear zones in outcrops and core drillings on active faults commonly reveal two scales of localization, with centimeter to tens of meters thick deformation zones embedding much narrower zones of $\mathrm{mm}$ - to $\mathrm{cm}$-scale. The narrow zones are often attributed to some form of fast instability such as earthquakes or slow slip events. Surprisingly, the double localisation phenomenon seem to be independent of the mode of failure, as it is observed in brittle cataclastic fault zones as well as ductile mylonitic shear zones. In both a very thin layer of chemically altered, ultra fine grained ultracataclasite or ultramylonite is noted. We present an extension to the classical solid mechanical theory where both length scales emerge as part of the same evolutionary process of shearing the host rock. We highlight the important role of any type of solid-fluid phase transitions that govern the second degree localisation process in the core of the shear zone. In both brittle and ductile shear zones chemistry stops the localisation process caused by a multiphysics feedback loop leading to an unstable slip. The microstructural evolutionary processes govern the time-scale of the transition between slow background shear and fast, intermittent instabilities in the fault zone core. The fast cataclastic fragmentation processes are limiting the rates of forming the ultracataclasites in the brittle domain, while the slow dynamic recrystallisation prolongs the transition to ultramylonites into a slow slip instability in the ductile realm.
\end{abstract}

\subsection{Introduction}

The geologist in the field is often confronted with two scales of localisation when investigating shear or fault zones[CHE 98, BEN 03]. Brittle fault zones show striking examples of extremely localized slip events, occurring within a thin shear zone, $<$ $1-5 \mathrm{~mm}$ thick, called the principal slipping zone (PSZ) [SIB 03]. This localized PSZ lies within a finely granulated fault zone of typically tens to hundreds millimeter

Chapitre rédigé par Manolis VEVEAKIS. 
thickness. These fault zones are either cataclastic in the brittle regime or mylonitic in the ductile regime.

Exhumed field examples for the morphology of these brittle fault zones can be found in the North Branch San Gabriel fault [CHE 93], the Punchbowl fault of the San Andreas system in southern California [CHE 98], the Median Tectonic Line fault in Japan [WIB 03], and in the Hanaore fault in southwest Japan [NOD 05]. Active faults have been intersected in wells in e.g. the Aigion system, central Greece, where the fault zone of clay size particles consists of finely crushed radiolarites, extended to about $1 \mathrm{~m}$. This localized zone of deformation was found to be intercepted by a "fresh"distinct slip surface of sub-millimiter size [SUL 07, COR 04]. Other wells that have been specifically designed to intersect active faults are the completed San Andreas Fault project [HOL 11] and the ongoing Alpine Fault drilling project [TOW 09]. The San Andreas Fault project revealed a fault zone of 1-2.5 m width at $3 \mathrm{~km}$ depth, with several ultralocalized PSZ's.

Similarly, in the ductile field the dynamic recrystalization of the matrix minerals is telltale of crystal plastic or diffusion creep, forming shear zones of vast thickness interrupted by ultralocalized anastomosing patterns of extreme grain size reduction in the ultramylonite. Exhumed thrusts in creeping carbonates around the world like the Naukluft thrust in Namibia [ROW 12], the McConnell thrust in Alberta Canada [KEN 97] or the Glarus Thrust in Switzerland [HER 08], all present a common structure of a meter-wide ( $1-5$ meters) zone which accomodates several thin $(\mathrm{mm}-\mathrm{cm}$ at most) veins of ultralocalized deformation and chemical alteration.

The brittle field features micro-mechanisms, governed by grain breakage, rolling, cleavage and brittle fragmentation processes. The ductile field features dislocation, diffusion and dissolution mechanism. Although these mechanisms are completely different they surprisingly exhibit he same dual pattern of localisation: a broad meter wide shear zone with ultra localised PSZ's in its middle.

Each of the different phenomena can be explained independently but to date no comprehensive theory has been suggested that can investigate the reason for the double degree localisation process and the potential commonalities between brittle and ductile localisation. This is because the dynamic process of the extreme localisation and the quasi static response of the background flow of the shear zone are treated as two different fundamental theories. The former instability is described in Solid Mechanics and the latter flow in Fluid Dynamics.

While Solid Mechanics is capable of accurately describing the conditions for failure it does not emphasise the long term post-failure behaviour. This is due to the nature of the solid materials that maintain a high degree of structural integrity after failure and are not significantly affected by the elapsed time. The consequence of this 
is that a quasi static approach is preferred thus making it possible to cast the weak rate effects into approximate laboratory determined hardening laws.

Fluid Dynamics on the opposite does not have the concept of failure, it knows nothing about the processes that happen prior to failure and is totally concerned with the description of the rate of flow of the material under an applied stress. Since most mantle convection codes are based on the fluid dynamical approach, the geophysical community has over the recent years expended a lot of effort into incorporating the theory of Solid Mechanics.

The fact that both theories apply to geology has been universally acknowledged and the after dinner contribution of Reiner on the 'Deborah Number" [REI 64] is well worth reading. The number is defined as the ratio of the stress relaxation time over the time of observation. Reiner's quote "the mountains flowed before the lord" implies that given enough time one may smoothly transition from one to the other theory. This can be easily conceptionalized in a Newtonian (linear viscous) viscoelastic framework, however, the inclusion of non-linear temperature dependence of the creep processes gave rise to the Time-Temperature Superposition (TTS) in polymer sciences [TOB 45]. The TTS principle implies that the temperature dependent behaviour of materials can be substituted by appropriate experiments at different time-scales for a reference temperature. It is thereby assumed that the time evolution described in the temperature equation is the only time-scale relevant for the long term mechanical behaviour of solids. This simple concept allows us to directly extend it for the nonlinear viscous behaviour of solids within a single theoretical framework incorporating elasticity, plastic failure and viscous post failure evolution.

In the present work we summarise a theory that couples the solid and fluid-like behaviour. This theory must be able to provide the framework for modeling both the formation of faults (refer, for more in depth reading, to [REG 13a, REG 13b] ) and their post-failure evolution. We show that the formation and post failure evolution of faults depend strongly on the coupled multiphysical effects affecting pressure, temperature and chemical conditions. We thus aim at providing in this paper a comprehensive guide through the energetics of faults and explain how they apply to the different types of failure.

\subsection{Time-independent formation of shear zones from Solid Mechanics}

The theory describing the onset of localized failure from uniform deformation has been a direct extension of the classic Mohr's theory of the strength of materials , and is applied to geomaterials using the so-called Thomas - Hill - Mandel shear-band model [HIL 62, MAN 66], which was introduced in the early 60s. A seminal paper was contributed by Rudnicki and Rice [RUD 75]. More recently, the mathematical formulation of bifurcation and post-bifurcation phenomena and related instabilities 
were summarized in [VAR 95] to form the basis of an improved continuum theory of failure of geomaterials.

This theory defines failure as stationary elastic wave and does not consider time evolution as a degree of freedom. While velocity dependent solutions are sought, they are independent of their evolution in true time. This is known as quasi-static deformation. The fundamental stress and strain solutions therefore degenerate into a geometric problem where failure lines appear with a finite width $d$.

These concepts of shear banding as material bifurcation of an elasto-plastic skeleton have led to the identification of a material length scale defining the width of shear bands. The critical stress, as well as the orientation and the thickness of the localized shear failure planes (shear zones) are calculated through the eigenvalues of the elastoplastic stiffness modulus $C_{i j k l}^{e p}$ of the material [RUD 75] obeying a rate-independent rheology, $\dot{\sigma}_{i j}^{\prime}=C_{i j k l}^{e p} \dot{\epsilon}_{k l}$ (the prime denoting effective stress). Within the framework of the solid mechanical instabilities the shear (fault) zone thickness emerges as a solution depending on the microstructure [MUH 87, PAP 11].

This rate-independent regime is typically used as an adequate descripion of brittle processes, thus placing the formation of the shear zones (onset of localization) near the maximum deviatoric stress [RUD 75]. For example, in linear elastic fracture mechanics brittle fracture occurs without thermal activation when a critical stress level is reached to split the bonds. At a critical energy threshold an elastodynamic fast time scale instability ensues where a variety of dissipative processes kick in such as grain/particle rotations which release heat in extremely fast timescales. The fast time scale instability simplifies the processes as temperature and fluids do not have time to diffuse. This regime is called undrained-adiabatic and is characterising extremely fast co-seismic slip [RIC 06, SUL 11] of brittle failure events.

Within this brittle, solid mechanical framework recent studies [SUL 11, VEV 12 , VEV 13] derived different levels of localisation corresponding to different energy (temperature) regimes. This result is a direct generalisation of the extension of the visco-elastic Deborah number concept to different temperatures using the time temperature superposition principle [TOB 45] for elasto-plastic media. This approach is generalising the concept of visco-elastic relaxation experiment at different temperatures to that of nonlinear visco-elasto-plastic solutions where quasi static solutions are sought for different temperatures.

In order to so we first freeze time by adopting classical, rate-independent, elastoplastic theory for a fault zone material incorporating chemical, hydraulic and thermal sensitivity. In the brittle field these fault zones are characterised by a damage zone surrounding the fault zone with an embedded PSZ as shown in Fig. 8 (a). In the brittle crust the width of this hierarchical damage zone can be up to 3-5 $\mathrm{km}$ wide [BEN 03, ALL 12], containing in principle multiple localization zones [FAU 03]. In order to 
keep the mathematical model tractable, we restrict the present work to the study of a single fault zone.

We therefore calculate the extent (thickness) of the brittle fault zone at different temperature regimes, marked by: (1) the temperature inferred from geothermal consideration for the boundary of the fault zone, (2) the activation temperature of the dominant chemical reaction observed in the PSZ. The details of the approach can be found in [SUL 11, VEV 12, VEV 13] and the results are summarised in Figure 8 (b) and (c).

\subsubsection{Shear Zone Thickness at Boundary Temperature Conditions}

The general solution for a quasi static shear zone considers the average of the background particle size $d_{50}$, the fluid pressure and the dominant chemical reaction at a given temperature. Both fluid pressure and reaction rate are strongly affected by the temperature. At ambient geothermal temperatures of the shear zone the chemical reaction is inactive and the thickness of the shear zone is predominantly controlled by the thermal-elastic pressurisation of the poro-elastic skeleton (Fig. 8 (b)), characterized by the pressurization coefficient $\Lambda=\frac{\alpha_{f}-\alpha_{s}}{K_{s}+K_{f}}(\mathrm{MPa} / \mathrm{K})$. In this expression, $\alpha$ is the thermal expansion coefficient and $K$ the compressibility, with subscripts $s, f$ denoting solid and fluid phases, respectively.

The solid mechanical thickness of the shear zone can be calculated directly as function of the average particle size $d_{50}$, and the thermal pressurisation $\Lambda$ as well as a multiplier $C$ representing the elasto-plastic material:

$$
\frac{d}{d_{50}}=\sqrt{\frac{C}{\Lambda}}
$$

For a Drucker-Prager, Cosserat material it was shown by [VEV 13, RAT 17a, RAT 17c, RAT 17b] that $C=17.5 \cdot 10^{3} \mathrm{KMPa}^{-1}$. For typical values of $\Lambda$ between $10^{-4}(\mathrm{MPa} / \mathrm{K})$ [CEC 11] and $1(\mathrm{MPa} / \mathrm{K})$ [RIC 06], with $d_{50}$ of the order of $1 \mathrm{~mm}$, the shear zone width ranges between 0.1 and 13 meters.

\subsubsection{Shear Zone Thickness at Elevated Temperature}

The effect of chemical reaction can be incorporated by considering the reaction rate to follow Arrhenius kinetics. In this framework the reaction rate depends on the activation enthalpy $Q_{F}$ and the reaction rate frequency $k_{F}$. The reaction is activated at elevated temperatures, near the activation temperature of the reaction at given pressure conditions. At these temperature conditions, and considering calcite decomposition as a typical reaction for a carbonate host rock [FAM 08, SUL 09], the thickness of the PSZ is several orders of magnitude smaller than the low temperature shear zone as shown in Fig. 8 (c). 
This result is reconciling field observations from two different temperature regimes but has no predictive power on how these temperatures may have been achieved. The traditional concept of TTS is not extendable to obtain extended solution for non-linear solids. We therefore need a non-linear superposition that allows fault zone evolution through time. The non-linear concept in this work is introduced by a Taylor expansion of the plastic strain rate, accounting for higher-order terms describing the nonlinearities.

In Geomechanics several studies have been devoted to the thickness of shear bands in soils. Under purely mechanical effects the thickness of the initial shear band (with thickness $D$ in Figure 8 ) in a granular medium is around $16 d_{50}$ with $d_{50}$ the average $(50 \%$ weight $)$ grain diameter [MUH 87]. This zone appears to be too wide to be considered as the PSZ [RIC 06]. In principle the PSZ represents a very fine shear localization, consisting of ultrafine particles that have usually undergone mechanochemical degradation (gelification, decarbonation and dehydration reactions, melting, as thoroughly discussed by [TOR 11]). Therefore, the initial mechanism such as the shear band formation from homogenous deformation [MUH 87] is a consequence of the solid mechanical failure while the formation of PSZ's may be considered an effect of the fluid-like deformation following the onset of the initial structure.

Such post-failure behaviour may for instance be significantly influenced by the presence of fluids interacting with the rock, hence inducing coupled effects including shear heating and pore fluid pressurization. These mechanisms are therefore expected to control the strength during fault slip [SIB 73, LAC 80, WIB 05] and the weakening effect displayed in catastrophic landslides [VAR 2a, VEV 07, L. 07, BLA 08, GOR 10]. Thermo-poro-mechanical couplings due to shear heating [SUL 05, RIC 06] can be also associated to chemical effects such as dehydration of minerals or decomposition of carbonates, theoretically studied recently by [BRA 10, BRA 11b, SUL 09, VEV 10] and reported to take place in real faults [HIR 07] and experiments at laboratory conditions [HAN 07, FER 10, BRA 11a, PAO 11, COL 13]

\subsection{Time-dependent evolution of shear zones}

To this end, we assume a smooth function of the effective stress, temperature $T$ and additional internal variables $\xi$, expressing the constitutive behaviour of the material

$$
\dot{\epsilon}_{i j}^{p}=f\left(\sigma_{i j}^{\prime}, T, \xi\right)
$$

The temperature and $\xi$ obey their own evolution laws, namely the energy balance for $T$ and experimentally deduced evolution laws for $\xi$. By expanding Eq. (15) around the effective yield stress $\sigma_{Y}^{\prime}$ we obtain

$$
\dot{\epsilon}_{i j}^{p}=f^{\prime}\left(\frac{\bar{\sigma}_{i j}}{\sigma_{n}^{\prime}}\right)+\sum_{m \geq 2} f^{(m)}\left(\frac{\bar{\sigma}_{i j}}{\sigma_{n}^{\prime}}\right)^{m}
$$



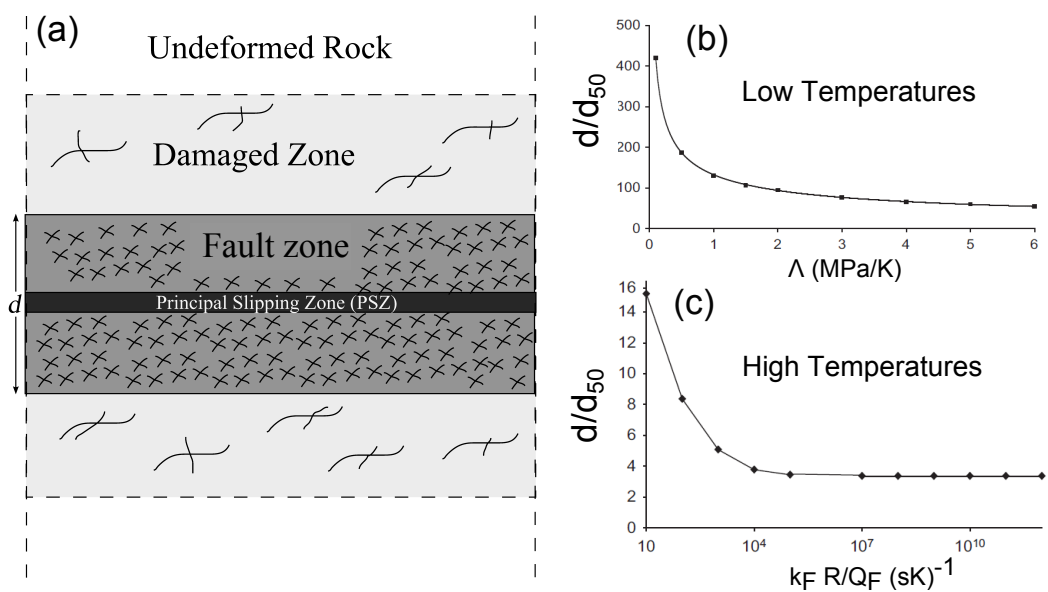

Figure 8. (a) Conceptual model of the internal structure of a fault described by two scales: the Principal Slip Zone (PSZ) in the centre surrounded by the fault zone. (b) using the geothermal temperature as a thermal condition for the boundary of the fault zone we obtain the thickness of the fault zone as a function of its internal structure (average particle size) and the thermal pressurisation. (c) Using the higher temperature required for chemical reactions as recorded in the PSZ a much length scale down to 3 times the average grain/particle size is obtained.

where $\bar{\sigma}_{i j}=\sigma_{i j}^{\prime}-\sigma_{Y}, \sigma_{n}^{\prime}$ a reference stress and $f^{(m)}=\frac{1}{m !}\left|\frac{d^{m} f\left(\sigma_{i j}^{\prime}, T, \xi\right)}{d \sigma_{i j}^{\prime m}}\right|_{\sigma_{i j}^{\prime}=\sigma_{Y}^{\prime}}$.

The first order of the Taylor expansion corresponds to the time independent solid mechanical regime presented earlier and describes the formation of the fault near initial yield. Once the fault has been formed after initial yield it continues to deform plastically (irreversible deformation) upon continuation of loading. This restricts our modelling efforts to the behaviour of the material inside the solid mechanical shear zone obtained from the first order expansion. Therefore, by considering explicitly the rate sensitivity emerging from the higher order terms of the expansion we study the non-linear (fluid-like) evolution of the mechanisms inside the solid mechanical fault zone solution.

The higher order terms of Eq. (16) give a natural extension of the classical TTS in the nonlinear visco-plastic space. If the solid mechanical yield is considered as a large scale reference state of our model we can describe the higher order terms in the nonlinear fluid dynamic space by the so-called overstress $\bar{\sigma}_{i j}=\sigma_{i j}^{\prime}-\sigma_{Y}^{\prime}$, defined as the stress that follows the evolution post the initial yield point [PER 66], hence 
$\bar{\sigma}_{i j}>0$ is always satisfied. The rate of plastic strain is therefore expressed as

$$
\dot{\epsilon}_{i j}^{p}=\sum_{m} f^{(m)}\left(\frac{\bar{\sigma}_{i j}^{\prime}}{\sigma_{n}^{\prime}}\right)^{m} \text { when } \bar{\sigma}_{i j}>0
$$

where $f^{(m)}$ has now dimensions of $(s)^{-1}$, being thus a reference strain rate.

In this sense the nonlinear TTS expresses a straight forward extension of the linear TTS with the difference that the time scale now plays an explicit role. In the classical TTS the time scale is simply derived from relaxation experiments returning the system near to its equilibrium whereas in the nonlinear TTS the system is always driven far from equilibrium. In the nonlinear framework the relaxation experiments therefore must be replaced by far from equilibrium energy considerations which define the time scales over which the rate of processes exchange energy inside the fault zone [REG 09].

\subsubsection{Energy considerations}

We introduce the Helmholtz free energy $\psi$, being a function of the elastic strain, temperature and the internal variables $\xi$. The energy balance equation, together with the second law of thermodynamics and Fourier's law of diffusion provide the local form of the entropy production equation [ROS 00, REG 09]:

$$
\frac{\partial T}{\partial t}+v_{k}^{m} \frac{\partial T}{\partial x_{k}}=c_{t h} \frac{\partial^{2} T}{\partial x_{k}^{2}}+\frac{q_{e}}{(\rho C)}+\frac{\Phi_{m}}{(\rho C)}+\frac{L}{(\rho C)}
$$

where $v_{k}^{m}$ is the barycentric velocity of the system, $c_{t h}=\frac{k_{t h}}{(\rho C)}$ is the thermal diffusion coefficient of the mixture (usually depending weakly on temperature and strongly on the microstructure, here considered constant), $C=-T \frac{\partial^{2} \psi}{\partial T^{2}}$ is the specific heat capacity of the mixture under constant volume, $q_{e}=\rho_{m} T \frac{\partial^{2} \psi}{\partial T \partial \epsilon_{i j}^{e}} \dot{\epsilon}_{i j}^{e}$ is the thermoelastic rate of heating. Note that in the framework of solid mechanics the advective terms are neglected, thus $v_{k}^{m}=0$.

The term $L=\rho_{m} T \frac{\partial^{2} \psi}{\partial T \partial \xi} \dot{\xi}=\Delta h r$ represents the latent energy produced or absorbed during a higher order energy transition ( $\Delta h$ is the enthalpy of the energy transition and $r$ its rate). This includes any microstructural changes such as grain size reduction and damage in the case that the state variable is a grain size or a damage parameter [LYA 14b, LYA 14a], respectively. In the case where the state variable refers to the chemical constituents it corresponds to the well known latent heat of a phase change. Finally $\Phi_{m}$ is the local dissipation, i.e. the mechanical power that is converted into heat in the system. In generalised thermodynamics the dissipation is defined by the product of a thermodynamic force times a thermodynamic flux. In mechanics the thermodynamics force is the stress $\sigma_{i j}^{\prime}$ and the thermodynamic flux is the plastic strain 
rate $\dot{\epsilon}_{i j}^{p}$. In the generalised framework, when additional state variables/processes are considered the force becomes a generalised force such as a damage force $E_{\xi}$ and the thermodynamic flux is the rate of change of the state variable $\dot{\xi}$.

$$
\Phi_{m}=\sigma_{i j}^{\prime} \dot{\epsilon}_{i j}^{p}-E_{\xi} \dot{\xi}=\chi \sigma_{i j}^{\prime} \dot{\epsilon}_{i j}^{p} .
$$

with $E_{\xi}=\rho_{m} \frac{\partial \psi}{\partial \xi}$ the energy dual of the internal variable $\xi$. The Taylor-Quinney ratio $\chi$ expresses the amount of the mechanical energy converted into heat [TAY 34], and is in principle a history dependent quantity rather than a constant $(0<\chi<1)$. Its importance is discussed in the following section.

\subsubsection{The Taylor-Quinney coefficient}

The Taylor-Quinney coefficient is a function of the elastic strain, the temperature and the internal variables:

$$
\chi=\chi\left(\epsilon_{i j}^{e}, \xi, T\right)=1-\frac{E_{\xi} \dot{\xi}}{\sigma_{i j}^{\prime} \dot{\epsilon}_{i j}^{p}} .
$$

The Taylor-Quinney coefficient is unity if all deformation work is converted into heat. For values smaller than unity and larger than zero it describes the portion of deformation work that is stored in microstructure. The coefficient can be derived through thermographic deformation experiments [ROS 00]. The Taylor-Quinney coefficient as derived from thermography often starts at initial yield being close to zero and progressively evolves in the transient deformation regime towards a steady state value of 0.9 for most materials [CHR 92]. Its evolution between these two values is therefore prescribed by the development of the internal state variables. As such it has a fundamental meaning for discriminating deformation mechanisms.

If the dissipation process is for instance a rapid fracture the evolution time of the Taylor-Quinney is extremely fast and obtained from the bond energy between the covalent or electronic bonds of the atoms. This equates macroscopically to an elastic interaction potential. In linear elastic fracture mechanics brittle fracture occurs at explosive timescales (of the order of seconds). The Taylor-Quinney coefficient prior to fracture is zero as all mechanical work is stored elastic deformation. At the critical threshold an elastodynamic fast time scale instability ensues where a variety of dissipative processes kick in such as grain/particle rotations which release heat therefore increasing the Taylor Quinney coefficient. This is the typical mechanism for brittle fracture.

Similarly in ductile deformation processes the Taylor-Quinney coefficient is zero at initial yield, however, in contrast to brittle deformation the failure is thermally activated. A series of micro-mechanical processes such as dislocation and diffusion 
mechanisms kick in that feature a time scale that is relatively slow compared to the elastodynamic time scale. The thermal activation is often described by an Arrhenius temperature activation. This rate-dependent failure mechanism is known as ductile (or creep) fracture.

$$
\Phi_{m}=\Phi_{0} \chi(\xi)\left(\frac{\bar{\sigma}_{i j}}{\sigma_{n}^{\prime}}\right)^{m+1} e^{-\frac{T_{0}}{T}}
$$

where $\Phi_{0}=\sigma_{n}^{\prime} \dot{\epsilon}_{0}$ is the reference dissipation, $\sigma_{n}^{\prime}$ is a reference stress value and $T_{0}=Q_{d} / R$ the thermal sensitivity (activation temperature) of dissipation.

\subsubsection{Chemical reactions}

From the analysis of the brittle regime (Section 2) we obtained that chemical reactions have a critical role in the system, defining the ultimate width of localization (the PSZ thickness). In this work we will emphasize on fluid release reactions, due to their fundamental importance in earth systems. Fluid-release reactions occur when either a hydrous mineral such as a clay mineral, serpentinite, mica, gypsum etc. looses its water at a critical activation enthalpy or when a mineral that is made of fluid phase and a solid constituent breaks down such as carbonate breaking to lime plus carbon dioxide upon critical activation enthalpy [FAM 08, SUL 09].

We treat these shear zone minerals as generalized solids characterised by the bonded chemical species $A$ and $B$ forming the solid composite $A B$. We assume $B$ to represent fluid species filling the porous matrix of $A B$ at initial conditions. At high temperatures the solid $A B$ breaks down, producing excess $B$ fluid, and increasing the fluid pore pressure through a general fluid-release reaction of the form $\nu_{1} A B_{(s)} \rightleftharpoons$ $\nu_{2} A_{(s)}+\nu_{3} B_{(f)}$.

The kinetics of this reaction are assumed to obey the standard Arrhenius dependency on temperature (see appendix .1). Following these considerations, the rates of the forward $\left(r_{F}\right)$ and reverse $\left(r_{R}\right)$ first order reactions (and for $\nu_{1}=\nu_{2}=\nu_{3}=1$ ) can be calculated to be

$$
\begin{array}{r}
r_{F}=\frac{\rho_{A B}}{M_{A B}}(1-\phi)(1-s) k_{F} e^{-Q_{F} / R T} \\
r_{R}=\frac{\rho_{A} \rho_{B}}{M_{A} M_{B}}(1-\phi) s \Delta \phi_{c h e m} k_{R} e^{-Q_{R} / R T}
\end{array}
$$

The total reaction rate is subsequently

$$
r=\left[(1-s)-s \Delta \phi_{\text {chem }} \frac{\rho_{A} \rho_{B}}{\rho_{A B}^{2}} \frac{M_{A B}^{2}}{M_{A} M_{B}} K_{c}^{-1} e^{\Delta h / R T}\right](1-\phi) \rho_{A B} k_{F} e^{-Q_{F} / R T}
$$


where $K_{c}=k_{F} / k_{R}$ and $\Delta h=Q_{R}-Q_{F}$. In these expressions $k_{F}, k_{R}, Q_{F}, Q_{R}$ are the pre-exponential factors and activation enthalpies of the forward and reverse reaction, respectively and $M_{i}$ and $\rho_{i}$ is the molar mass and density of the $i-$ th constituent. The porosity $\phi$ consists of an initial value $\phi_{0}$ and the new interconnected pore volume created from the reaction $\Delta \phi_{\text {chem }}, \phi=\phi_{0}+\Delta \phi_{\text {chem }}$. The partial solid ratio $s$ is the volume ratio of the produced solid $A$ in the solid matrix. The expressions for the dependency of $\phi$ and $s$ on the reaction kinetics are given in [ALE 14] and are summarized here in appendix .1. This formulation is essentially a damage mechanics formulation with porosity being the damage parameter being controlled by the physics of the chemical reactions.

\subsection{Post failure evolution of a shear zone}

We focus on the post failure evolution of a shear zone with thickness $d$, formed by material bifurcation (Fig. 9). Due to the small thickness of the shear zone compared to the thickness of the overburden, its momentum balance prescribes a constant stress profile across the shear zone, thus $\sigma_{y x}=\tau_{n}(t)$ and $\sigma_{y y}=\sigma_{n}(t)$ [RIC 06, VEV 10]. In addition, in the presence of a fluid, the stress can be decomposed according to Terzaghi's principle [VAR 95] to $\sigma_{i j}^{\prime}=\sigma_{i j}+p_{f} \delta_{i j}$, with $\delta_{i j}$ the Kronecker's delta and $p_{f}=p_{n}+\Delta p$ the pore fluid pressure, consisting of a hydrostatic part $p_{n}$ at the boundary and the excess pore pressure $\Delta p$. The final system of equations is obtained once the energy equation is coupled with the mass balance equation [VEV 10, ALE 14, VEV 14a, VEV 14b]:

$$
\begin{array}{r}
\frac{\partial \Delta p^{\star}}{\partial t^{\star}}=\frac{\partial}{\partial y^{\star}}\left[\frac{1}{L e} \frac{\partial \Delta p^{\star}}{\partial y^{\star}}\right]+\Lambda \frac{T_{c}}{\bar{\sigma}_{n}^{\prime}} \frac{\partial \theta}{\partial t^{\star}}+(1-\phi)(1-s) \mu_{r} e^{\frac{A r \theta}{1+\theta}} \\
\frac{\partial \theta}{\partial t^{\star}}=\frac{\partial^{2} \theta}{\partial y^{\star 2}}+\delta\left[G r\left(1-\Delta p^{\star}\right)^{m} e^{\frac{a A r}{1+\theta}}-(1-\phi)(1-s)+(1-\phi) s \Delta \phi_{\text {chem }} \eta K_{c}^{-1} e^{\frac{x A r}{1+\theta}}\right] e^{\frac{A r \theta}{1+\theta}}
\end{array}
$$

where the fields were normalised with the help of a reference temperature $T_{c}$ :

$$
t^{\star}=\frac{c_{t h}}{(d / 2)^{2}} t, y^{\star}=\frac{y}{d / 2}, \theta=\frac{T-T_{c}}{T_{c}}, \Delta p^{\star}=\frac{\Delta p}{\bar{\sigma}_{n}^{\prime}}
$$

The kinetics of the reactions are normalised using the Arrhenius scaling

$$
A r=\frac{Q_{F}}{R T_{c}}, a=1-\frac{Q_{d}}{Q_{F}}, x=1-\frac{Q_{R}}{Q_{F}}
$$


The remaining dimensionless quantities appearing in the system (24) are defined as

$$
\begin{array}{r}
\delta=\frac{|\Delta h|(d / 2)^{2}}{k_{t h} T_{c}} k_{F} \rho_{A B} e^{-A r} \\
\eta=\frac{\rho_{A} \rho_{B}}{\rho_{A B}^{2}} \frac{M_{A B}^{2}}{M_{A} M_{B}} \\
\mu_{r}=\left(\frac{\rho_{A B}}{\rho_{B}} \frac{M_{B}}{M_{A B}}\right) \frac{(d / 2)^{2}}{c_{t h} \bar{\sigma}_{n}^{\prime}} \frac{k_{F}}{\left(\beta_{f}+\beta_{s}\right)} e^{-A r} \\
L e=\frac{c_{t h} \mu_{f}\left(\beta_{f}+\beta_{s}\right)}{k} \\
G r=\frac{\chi(\xi) \Phi_{0}}{k_{F}|\Delta h|\left(\rho_{A B} / M_{A B}\right)}
\end{array}
$$

where $k_{t h}=c_{t h}(\rho C)$ the thermal conductivity of the system. The system of Eqs. (24) is fully described when the 8 dimensionless numbers $\left(a, x, \mu_{r}, L e, \delta, G r, K_{c}\right.$, and the boundary temperature $\theta_{b}$ since $A r$ is implicitly considered in these groups) are determined. However, the system has a much lower dimensionality which can be revealed through a comprehensive analysis of its steady state and transient responses. These analyses has been presented in [ALE 14, VEV 14a, POU 14a], identifying the dominant parameters of the system. Of critical importance is the Gruntfest number $G r$, as it incorporates the energetics of the microstructure through the Taylor-Quinney coefficient, as well as the mechanical loading and the characteristics of the chemical reaction. High values of $G r$ correspond to a regime where the mechanical input is sufficient to trigger the chemical reaction. Due to its fundamental nature, $G r$ will be treated as a bifurcation parameter.

The post-failure evolution of the shear zone is summarized in Fig. 10. The first panel (a) illustrates the fundamental three phase stability S-curve typical to all systems of the generalized type discussed above (Eq. 24) [YUE 77]. The first phase is illustrated in details in panel (b) showing a steady state creep solution acting as a global stable material response without instabilities. The panel (c) is the solution for the phase of elevated Gruntfest numbers but below the critical point B of Fig. 10 (a). For any initial conditions below line $\mathrm{BC}$ of (a) the system relaxes back to the stable branch $\mathrm{AB}$. For any initial condition above line $\mathrm{BC}$ of (a) the system features a solitary oscillation. Panel (d) of Fig. 10 illustrates the behaviour in domain III where a stable oscillator emerges. This stable oscillator is self-sustained and has a fundamental role in the post-failure evolution of shear zones. This area, where $G r>G r_{B}$, is the domain where localisation of dissipation occurs owing to critical energy transitions. This means that the latent energy term $L$ of Eq. 18 is triggered and energy is released. This domain does not depend on the exact micro mechanism, as shown in [VEV 10], 

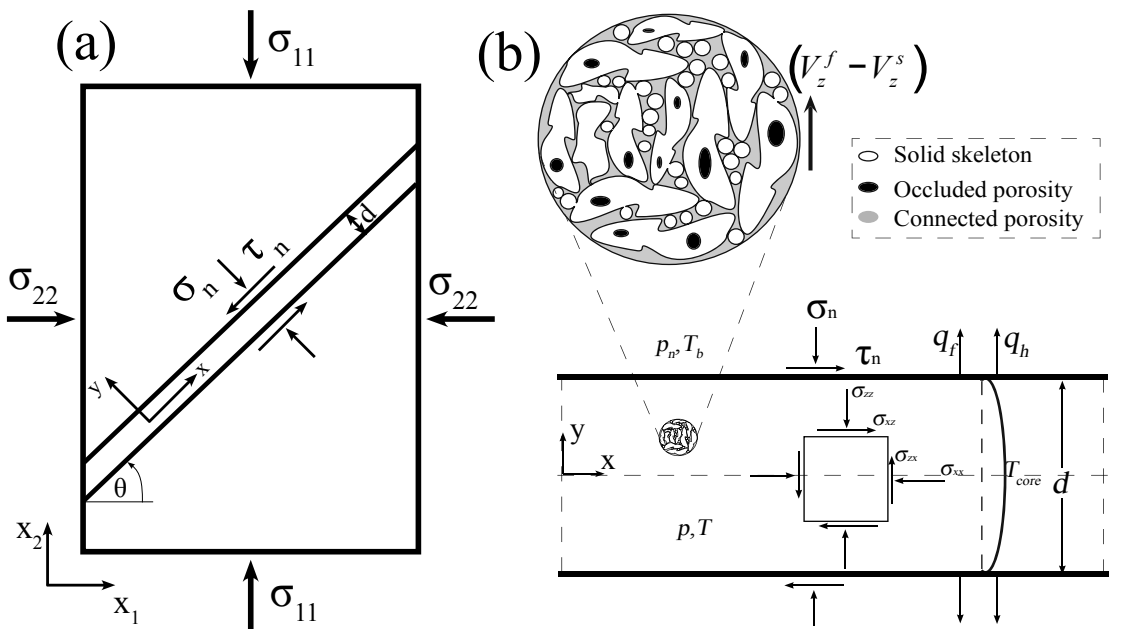

Figure 9. (a) Sketch of the continuum mechanical concept of shear failure as a material bifurcation [RUD 75]. At a critical stress ratio of the original stresses (in the coordinate system $x_{1}-x_{2}$ ) a conjugate pair of shear zones emerge, having a thickness $d$ and dipping at angles $\pm \theta$ thereby defining the rotated coordinate system of failure, $x-y$. For visual purposes only the shear zone dipping at $\theta$ is shown here. (b) Processes inside the shear zone of $(a)$. The loading conditions and the filter velocity $v_{z}^{f}-v_{z}^{s}$, along the content of a saturated rock, are depicted. We assume that any chemical reaction is taking place at the solid-pore interface. Hence all the produced fluid contributes to the interconnected pore volume and is concentrated on the grain boundaries. We also assume that the produced solid is added to the skeleton, establishing a common velocity field $v_{z}^{s}$ with the reactant solid.

but describes a generalised material instability where the changes of the state variable form the localisation phenomenon. The state variable can be for instance a grain size, a damage or porosity change as discussed in this study.

Alevizos et al. [ALE 14] provided asymptotic criteria for the area of oscillations to be admitted. This occurs when the following inequalities simultaneously hold:

$$
G r>\left(1+\frac{e^{-A r \theta_{b}}}{A r \delta}\right) e^{-a A r}, \mu_{r} L e<A r^{-3 / 2}, \log \left(K_{c}\right) \gg 1
$$

These inequalities correlate most of the dimensionless groups of the system, implying that in environments where strong (irreversible) endothermic reactions are favoured (hence $\log \left(K_{c}\right) \gg 1$ is always satisfied) any 2 of the remaining groups can always be selected as control over oscillatory instabilities. 


\subsubsection{Analysis of the system's response}

The solution discussed in Fig. 10 is obtained under the assumption of a constant loading stress and chemistry as well as internal microstructure thus giving a constant Gruntfest number. In the more general case either of these parameters can evolve in time thus allowing the system to cross all three areas of the phase diagram. This leads potentially to a multiplicity of chaotic responses marked by phases of self-organised chaos, transition from aseismic creep to seismic slip and back. For example if the system follows the hysteretic loop ABB'CA of Fig. 10 we find all three different system responses in one and the same shear zone.

Of particular interest for plate tectonic loading is the particular attractor depicted in Fig. 10 (d) where a constant plate velocity would lead to a constant shear stress over time, allowing for a steady state evolution of the microstructure and chemistry. The periodic instability that emerges in this regime corresponds to a stick-slip type of instability, as illustrated in Fig. 11 where the limit cycle is plotted in the logarithmic strain rate versus normalised time space. The limit cycle is characterised by two distinctly different time scale phenomena, the first being a long time scale of slow creep interrupted by a short time scale fast response owing to fluid the release reaction.

In addition to the two timescales characterizing the system's time evolution, its spatial manifestation also comprises two length-scales. Fig. 12 shows profiles of strain rate (red line), porosity (blue line) and solid product (green line) inside the solid mechanical shear zone, at their maximum points in the limit cycle. Starting from a flat initial profile when the solid mechanical shear zone is established, the strain rate localizes in an ultrathin core zone during the fast timescale. The extreme localisation of strain rate in the centre of the solid mechanical shear zone is a robust outcome for any chemical reaction. The thickness of this ultralocalized shear band inside the global mechanical shear zone depends on the activation enthalpy of the forward reaction. For large enthalpy reactions the localised zone is ultra-sharp and broadens with reducing enthalpy. This localisation is then followed by a chemicalmechanical localisation wave propagating towards the boundaries of the shear zone [VEV 15, VEV 14c, STE 14].

We expect from this result two important outcomes. The first is that the shear zone will show at least two different scales of localisation, linked to the corresponding timescales. The broad solid mechanical zone acts as a vessel for the long creeping timescale, whereas the sharp fluid-dynamic length-scale accompanies the fast pressurization timescale. The second outcome is that the width of the fluid dynamic localisation can be used to identify the activation energy of the chemical reaction involved. The spatial extent of the fluid release reaction is shown in the blue porosity curve in Fig. 12 to broadens with reducing enthalpy of the reaction. The same trend applies to the solid constituent of the reaction. This additional information may be used in the field as added constraint for identification of activation enthalpies. 


\section{non-peer reviewed EarthArXiv preprint}
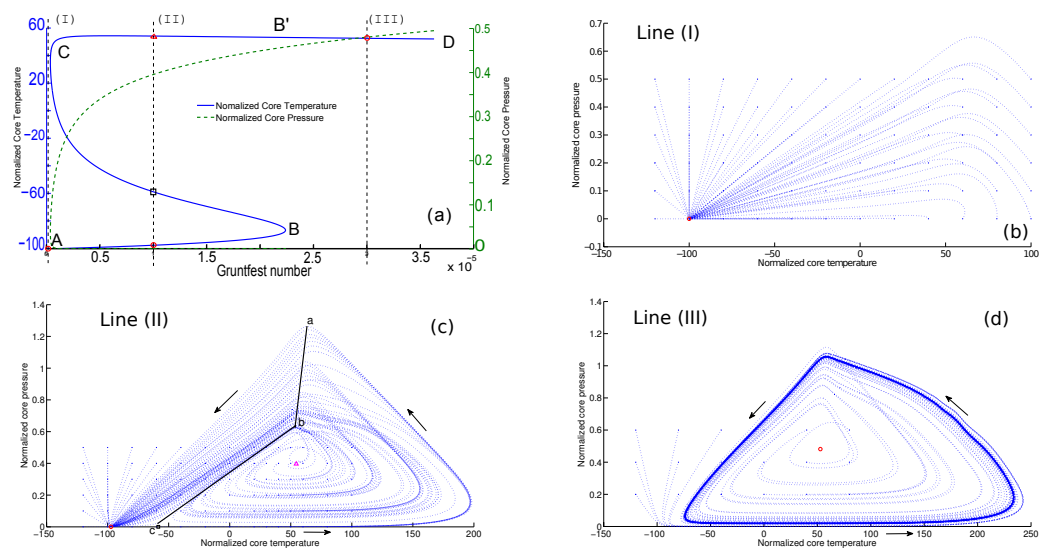

Figure 10. Summary of the system's response when Eqs. (28) are satisfied. (a) Steady state response of the dimensionless core temperature (solid line) and the dimensionless core excess pore pressure (dashed line). The three lines annotated correspond to the three areas of interest providing the phase diagrams of: (b) a stable, aseismic creep behavior (line I), where all the initial conditions in the $\Delta p-T$ phase plane end up at the stable node depicted as a circle, (c) non-periodic events (line II), where the line a-b-c separates the linear paths towards the lower stable node (circle) from the homoclinic orbits tending to the circle via the unstable spiral orbit of the upper solution (triangle), and (d) periodic instabilities (line III), appearing in the $\Delta p-T$ phase plane as stable limit cycles around the unstable upper steady state solution (circle). The magnitude of the cycle increases with decreasing Gr, obtaining its maximum amplitude at point $B$, where a homoclinic bifurcation takes place, with the periodic orbit colliding with the saddle point $B$. Note that values of $\Delta p$ larger than 1 indicate possible hydraulic fracturing in the vicinity of the fault's core, limiting the present model.

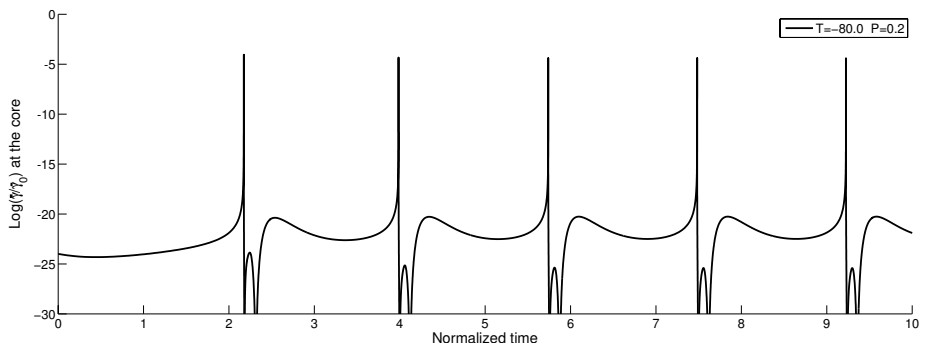

Figure 11. Evolution of the core strain rate on line III of Fig. 10 (a). Irrespectively of the initial conditions, the system in this area undergoes cycles of abrupt acceleration followed by prolonged relaxation. 

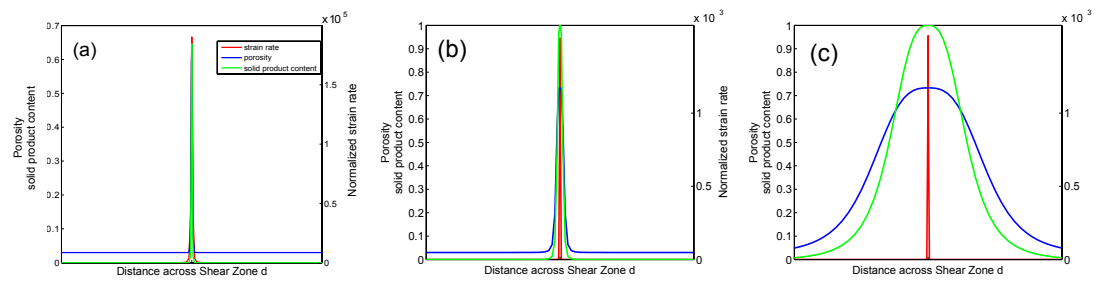

Figure 12. Influence of the characteristics of the reaction. Snapshots of the profiles of porosity $\phi$, solid product content and strain rate near the time of their maximum values (the point of maximum temperature in the limit cycle of Fig. 10d). (a) For $\Delta h=Q_{F}$, (b) for

$\Delta h=3 / 4 Q_{F}$, (c) for $\Delta h=Q_{F} / 2$. We notice that as $\Delta h$ decreases the reaction broadens its extent inside the shear zone $d$. For $\Delta h \approx Q_{F}$ porosity and s are weakly produced at the core of the shear zone, and the strain rate is smaller in magnitude (a). Once $s$ and $\phi$ reach their maximum values (in this case $s=1$ and $\phi=0.75$ ) the reaction takes place in an increasingly broader zone as $Q_{R}$ increases $(b-c)$.

\subsubsection{Timescales of the system}

We expand the temperature field with respect to $\epsilon=1 / A r$ [FOW 97]. At leading order the chemical reaction is inactive, thus reducing the system to a single equation, known as the Frank-Kamenetskii limit:

$$
\frac{\partial \theta}{\partial t^{\star}}=\frac{\partial^{2} \theta}{\partial y^{\star 2}}+\lambda e^{\theta}
$$

where $\lambda=\delta\left(G r e^{a A r}-1\right)$. Equation (29) is correspondingly the Frank-Kamenetskii equation [FUJ 69], known to have the semi-analytic solution [VEV 07]:

$$
\theta=\theta_{\text {core }}-\ln \left[\lambda\left(t_{I}-t^{\star}\right)+\frac{y^{\star 2}}{4\left(c_{1}-\ln \left(t_{I}-t^{\star}\right)\right)}\right]
$$

where $\theta_{\text {core }}$ is the initial temperature at the core (maximum), $t_{I}=1 / \lambda$ is the time that temperature presents a singularity (blow-up time), and the constant $c_{1}$ is determined by the boundary conditions [VEV 07]. We note that the initial condition should be the temperature at which the fault is initially formed. For flat, isothermal profile inside the shear zone, $\theta_{\text {core }}=\theta_{b}$.

As discussed in [VEV 07], past a critical strain rate achieved at $t \approx 0.88 t_{I}$, the analytical solution of Eq. (30) indicates that dissipation (strain rate) localizes towards the center of the shear band while abruptly increasing. When reaching the critical value of temperature to trigger chemical pressurization, then the system enters the pressurization regime and excess pore pressure is being generated from the reaction in an undrained adiabatic setting. The time at which the temperature at the center of the faul zone $(y=0)$ reaches the activation temperature of the reaction, $\theta_{c r}$, is 
approximately the time-scale of the frictional (stable creep under zero ovepressures) process,

$$
t_{c r}^{\star}=\frac{1}{\lambda}\left[1-e^{\left(\theta_{c o r e}-\theta_{c r}\right)}\right]
$$

Thus, the time at which chemical pressurization will set in is a function of the initial configuration of the system $\theta_{\text {core }}$, of the pressurization temperature $\theta_{c r}$ and of all the material, chemical and loading parameters of the problem, incorporated into $\lambda$. Recalling that $G r$ (hence $\lambda$ ) incorporates the Taylor-Quinney coefficient, $t_{c r}^{\star}$ is directly influenced by the evolution of the internal variables, thus of the microstructure.

Note that when $\theta_{c r}=\theta_{\text {core }}$ then $t_{c r}^{\star}=0$, meaning that the fault will enter the pressurization regime directly, without admitting any period of creep. On the other hand, when $\theta_{c r} \gg \theta_{c o r e}$, then $t_{c r}^{\star}=1 / \lambda$ and the fault will admit all its creeping capability. Once the chemical reaction is triggered it evolves in a fast timescale, estimated by the higher order of the expansion, where undrained-adiabatic conditions establish. In this regime, the timescale is inversely proportional to $\mu_{r}$ [VEV 10]

$$
t_{p}^{\star} \sim \frac{1}{\mu_{r}}
$$

\subsection{Comparison to Field Observations}

We have identified in the above theoretical considerations two fundamentally different processes (rate/temperature independent and rate/temperature dependent) with similar outcomes. Both processes lead to two scales of localisation. The large scale is associated with the solid mechanical solution and the small scale with the fluidised fault zone material inside the master shear zone.

In the rate/temperature independent solution the ultralocalised PSZ appears without a creeping phase. From Eq. (31) we conclude that the system is already in a critical state and does not require shear heating to be brought to criticality. In physical terms this could be seen as a transition that is equivalent to allowing phase changes, or grain size reduction, or damage to start at a negligible input of energy. This leads to the identification of the fast time-scale elastodynamic instability, where the energy released in the PSZ causes extreme localisation with an internal time scale governed by the energy change process. In our example we postulated a fluid release reaction and obtained the pressurisation time scale of Eq. (32). Other mechanisms, such as damage or grain-size, impose different time scales for instability based on their energetics. This dual localisation is therefore the hallmark of brittle shear zones as illustrated in Figure 13 (a).

In the case where the system is not close to criticality and is creeping the equivalent ductile localisation mechanism can emerge. Upon a finite time after release of 
deformational work into heat inside the creeping shear zone, the system can reach the critical point for a fast energy transition. At this point the creeping zone forms a PSZ upon which the micro mechanical or chemical changes of the fast energy process occur. We have discussed the example of a chemical breakdown reaction which results into an accelerated slip instability forming the PSZ. A number of other microstructural processes are often activated in the course of this instability leading to a rich microstructure inside the PSZ. This could be dynamic recrystallisation, fluid release, dissolution-precipitation etc. The complex nature of these instabilities leads to complex geometries and time series such as slow slip/earthquake signatures. The dual feature of creeping zone and ultralocalized PSZ, therefore is also a hallmark of ductile shear zones as illustrated in Figure 13 (b).

To juxtapose the outcomes of the theoretical approach with field evidence, we compare our solutions with observation from brittle and ductile shear zones. Figure 13 shows in (a) the famous Punchbowl cataclastic fault described by Chester and Chester [CHE 98]. The fault shows a cataclastic fault zone of the order of meter thickness with an ultralocalized, pulverised PSZ. The mineralogy of the host rock and PSZ indicate a transition from quartz and feldspar dominated to clay mineralogy in the cataclastic fault zone and ultimately higher ordered smectite and quartz in the PSZ[CHE 98]. This indicates a series of mineral dissolution-precipitation reactions that are typical for the ingression of water under lower temperature environments. The addition of water can decrease the activation temperature and thereby render the system critical at ambient conditions.

The same style of deformation is also recorded in the UNESCO world heritage Glarus fault that shows a meter-thick, chemically altered tectonite (known as Lochsite tectonite), blending the hanging wall and footwall minerals [POU 14b]. The tectonite is deformed an folded by ductile deformation and has an ultralocalised PSZ (Fig. $13 \mathrm{~b}$ ) in its middle. Field evidence include a multiplicity of PSZ's inside the tectonite, implying repetitive fast events interrupting ductile creep. The key chemical reaction controlling both the tectonite formation and the thickness of the PSZ was identified to be carbonate decomposition and precipitation [HER 08]. For the ductile localisation instability additional heat is required as the carbonate decomposition reaction happens at much larger temperature than the boundary of the thrust zone. The addition of temperature is readily available from the long-term dissipation of the creep process, which becomes a prerequisite for the ductile instability. Because the shear zone has a characteristic background temperature, the chemical reaction has a critical temperature to be triggered and the microstructure has equilibrated over time, to quasi-steady state, the ductile instability is inferred to have periodic signature in time. This implies a regular stick-slip type of behaviour for the ductile PSZ whereas the brittle PSZ is expected to be chaotic in time. The reason is that brittle instabilities are always at criticality while ductile instabilities require time to be brought to criticality. 

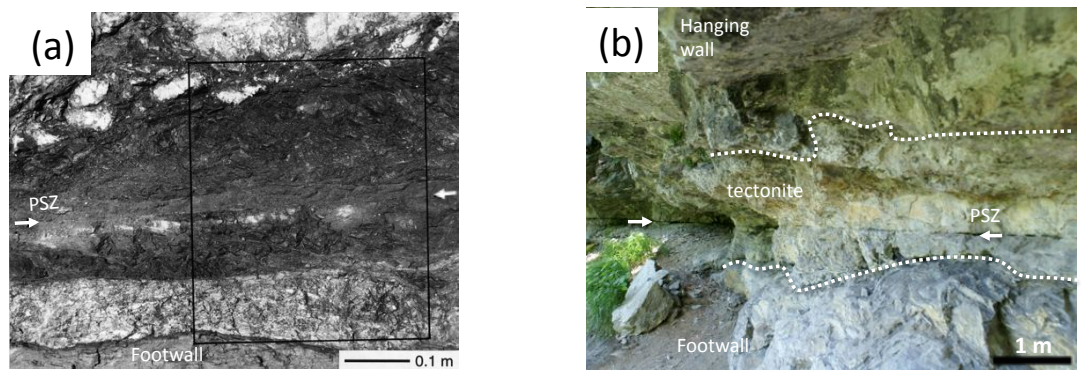

Figure 13. (a) Punchball fault from Chester and Chester [CHE 98] featuring two scales of localisation, the cataclastic fault zone and the ultracatlastic PSZ annotated by arrows. (b) The Glarus Thrust at the Lochsite, featuring two scales of localsation, the creeping zone forming the carbonate rich tectonite and the ultralocalized PSZ in its centre.

\subsection{Application to ETS sequences}

The temporal predictions of the model presented can be tested against real measurements from Episodic Tremor and Slip events (ETS) at the plate interface during the subduction of oceanic plates below the continental ones. In these subduction environments, key minerals controlling both the rheology and the fluid-release reaction are the minerals of the serpentinite family [POU 14a]. We may therefore specify the fluid release reaction to be the dehydration of serpentinite minerals. For the example of antigorite dehydration, $A B$ could correspond to antigorite $\left(\mathrm{Mg}_{48} \mathrm{Si}_{34} \mathrm{O}_{85}(\mathrm{OH})_{62}\right)$, $A$ would represent all the solid products such as olivine $\left(\mathrm{MgSiO}_{4}\right)$ and enstatite $\left(\mathrm{MgSiO}_{3}\right)$ and $\mathrm{B}$ the fluid $\mathrm{H}_{2} \mathrm{O}$. In this section, we use the values presented by [POU 14b] for the material properties of the system and study regular and irregular GPS signals from two different subduction environments.

\subsubsection{Regular sequences - Cascadia ETS sequence}

We begin with the periodically consistent case of the Cascadia ETS sequence in Canada (for a more detailed analysis refer to [ALE 14, VEV 14a, POU 14a]). Episodic transient movements are continuously recorded from a network of GPS sensors revealing consistent periodicity between slip events over 20 years. The magnitude of the slip events is about $10 \mathrm{~mm}$, being accumulated over approximately 2 weeks. We model the detailed displacement data available from the GPS stations in Vancouver Island [NAS ], exhibiting a period of 14 months between the slow slip events. Because of the nearly perfect periodic sequence, it is expected that the model will be able to predict the evolution with a single fundamental mode of oscillation. All the GPS data used in this section were de-trended from the background to obtain a zero average velocity. No further geophysical techniques were used to remove annual effects, so that the model can be juxtaposed against the raw signals. 
The model replicates the temporal sequence of the last 20 years, as shown in Fig. 14, highlighting the validity of the suggested interplay between dehydration reactions and the mechanical response. The inverted values of the parameters used [POU 14b] show that the serpentinite family minerals creep in an interface shear zone of about $6 \mathrm{~m}$ thickness in which the ETS sequence takes place.

The GPS data show some irregularities and offsets between the events, thus suggesting perturbations to the system due to seasonal or secondary transient effects. However, within the 20 years interval these perturbations are recovered by the system back to its fundamental mode, the stable multiphysical oscillator of fig. 14b. This in turn could suggest that there is small variation in the parameters and only one eigenmode is preferred. Thus, the system can absorb these perturbations due to the stable nature of the fundamental limit cycle and cannot switch to a different one.

In the case of Cascadia ETS sequence the loading conditions used ( $\tau_{n}$ and $\sigma_{n}$ ) were constant in time. Therefore, as suggested above, the 20 years of GPS recordings were matched with a single limit cycle of the system (Fig. 14b). This result reveals that Cascadia is a self-sustained process equilibrating near the minimum dissipation capacity of the subduction system.

\subsubsection{Alternating modes - Hikurangi ETS sequence}

A question that naturally arises is whether the suggested formulation can also be used for less regular GPS signals. The application of the present model to an irregular case study was performed by [POU 14b]. The basic assumption is that, unlike the Cascadia case, the existence of irregular ETS sequences can be attributed to non constant parameter values which forces the system to switch between different material oscillators. Such a case could be obtained if, for instance, the dimensionless groups are allowed to evolve in time (as shown in [POU 14a]) or in space, accounting for the different geologies and ambient conditions intersected by the fault. A natural consequence of the temporal and spatial variation of the parameters is that the system would turn into a multi-period, multi-scale chaotic attractor and the subduction system will depart from its minimum disspation limit and driven towards the maximum dissipation.

The Grunfest and the Lewis numbers are bearing the information of the loading conditions, and are therefore the parameters that can vary in time. Having already pointed out the role of the critical Gruntfest number $G r_{B}$ as a global attractor of the oscillator, we assume here that its evolution is the most probable cause for the presence of alternating oscillatory modes in the system. Following the discussion in section ?? the time variation of the Gruntfest and associated Lewis number can be affected by short term seismic stress perturbations in the vicinity of the subduction zone. As pointed out in the case of Cascadia, the fast time scale of the process is 

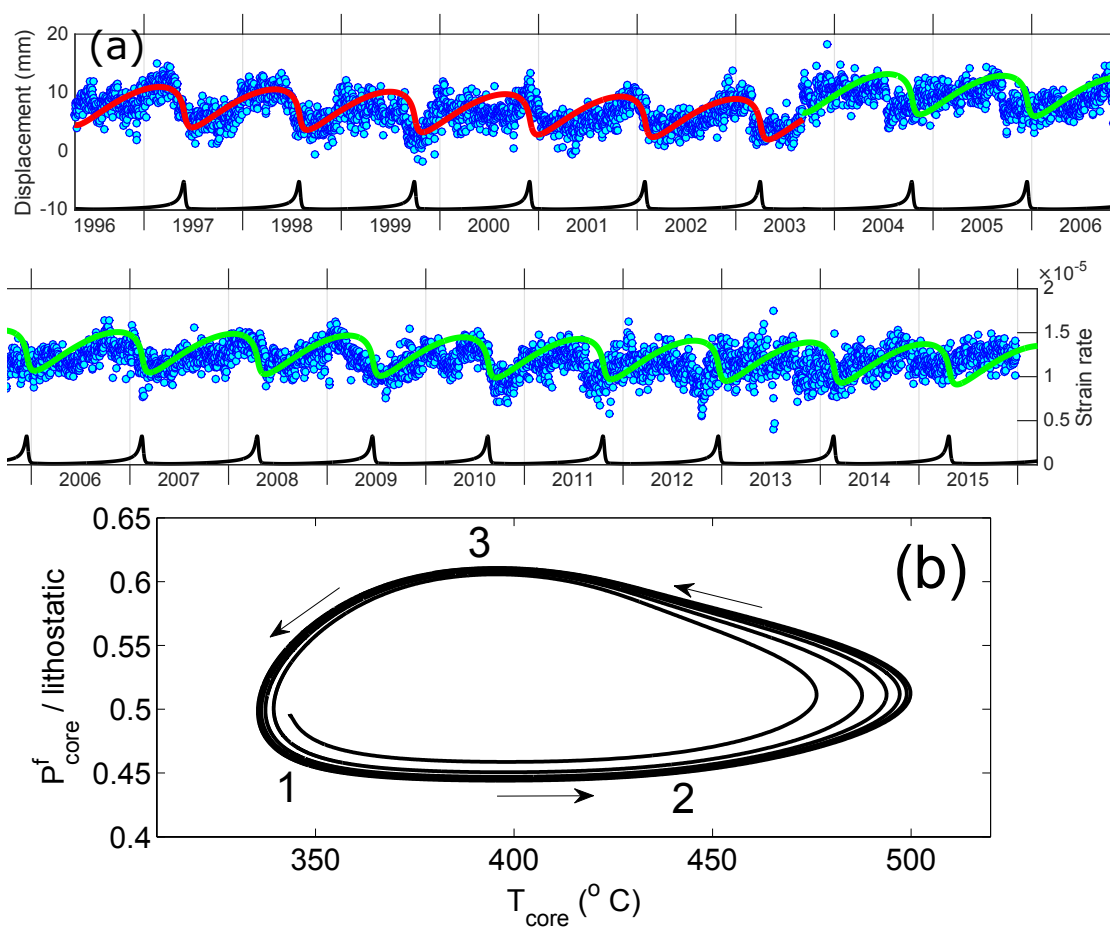

Figure 14. Regular ETS sequence in South Vancouver Island, Cascadia, station ALBH [NAS ]. (a) The GPS data (blue dots) represent raw displacement data with its linear trend removed. The continuous sequence is shown split into the period from mid-1996 to mid-2006 (upper row) and beginning 2006 to end-2015 (lower row). The 20-year ETS sequence is matched (red and green lines) by a single stable limit cycle (b) and the strain rate curve (black line) incorporates the long slow creep periods (path 1-2) as well as the slow earthquake events due to the fluid release during the reaction (path 2-3). Any perturbations are recovered bringing the system back to the same cycle, as reflected in the GPS data of $(a)$. The perturbation of mid-2000 was recovered by the system in 2001 and the perturbation, which affected the system in 2008, was recovered in 2011. Note that in October 2003 the sequence was interrupted due to a jump of the GPS sensor. The numerical fit is shifted (green line) to account for this jump. 
approximately 2 weeks. This means that a seismic event happens on an even faster time scale, which will provoke an almost instantaneous jump in the stress applied on the shear zone boundaries. The latter can be equated to step-like perturbations on the Gruntfest number in the framework of the present chemical oscillator model.

From the fitting process of the GPS sequence in New Zealand (Fig. 15) it was deduced that the oscillators comprise two modes. The first mode (mode 1 in Fig. 15) is exactly identical to the one derived from the Cascadia fit. The second mode is shown as mode 2 in Fig. 15 and has a significantly larger temperature variation of around $270^{\circ} \mathrm{C}$, from $330^{\circ} \mathrm{C}$ to approximately $600^{\circ} \mathrm{C}$. During both cycles the fluid pressure rises from hydrostatic values ( $40 \%$ of the lithostatic) to about $60 \%$ of the lithostatic when the forward reaction is the dominant mechanism. A major outcome of this fitting process is that, similar to Cascadia, the width of the shear zone where all the mechanisms presented operate, is calculated around 6 metres wide. In addition, as suggested in [POU 14b], the common ocsillator is a fundamental mode (or eigenmode in a mathematical context) of serpentinite deformation.

As far as material constants are concerned, these were considered to be the same for Cascadia and Hikurangi suggesting the common serpentinite nature of the lubricating shear zones. The only difference between the two cases is the emergence of a second oscillator in New Zealand that was attributed to an overstress transient of the order of $60 \mathrm{kPa}$ in shear [POU 14b]. This derivation suggests that the elastodynamic transients of the surrounding environment cannot be neglected in the Hikurangi trench. Since mode 2 displays the higher amplitude oscillations it is identified as the one closer to the global attractor (the homoclinic point) and mode 1 is interpreted as a higher Gruntfest number mode.

This means that in Hikurangi the dynamics of the serpentinite oscillator between the two modes depicted in (Fig. 15) are driven by evolving (in time) boundary conditions. Unlike Cascadia, where constant stress boundary conditions could reproduce 20 years of ETS activity, in New Zealand the loading stress exhibits time variations thus not placing the subduction environment in the minimum dissipation limit. However, since the system is is not admitting damped oscillations, Hikurangi it is also not driven at its maximum dissipation limit. It is therefore in an intermediate loading regime, oscillating continuously between 2 different modes and driving both the slow earthquake activity and the fast seismic events in the vicinity of Gisborne [?, see also]]Poulet2014a.

\subsection{Discussion}

In this contribution we link the observed double localization patterns in fault zones with a discussion on their possible driving energetics and timescales of formation. We have shown that the thick fault zone is a result of a solid mechanical instability, acting 


\section{non-peer reviewed EarthArXiv preprint}
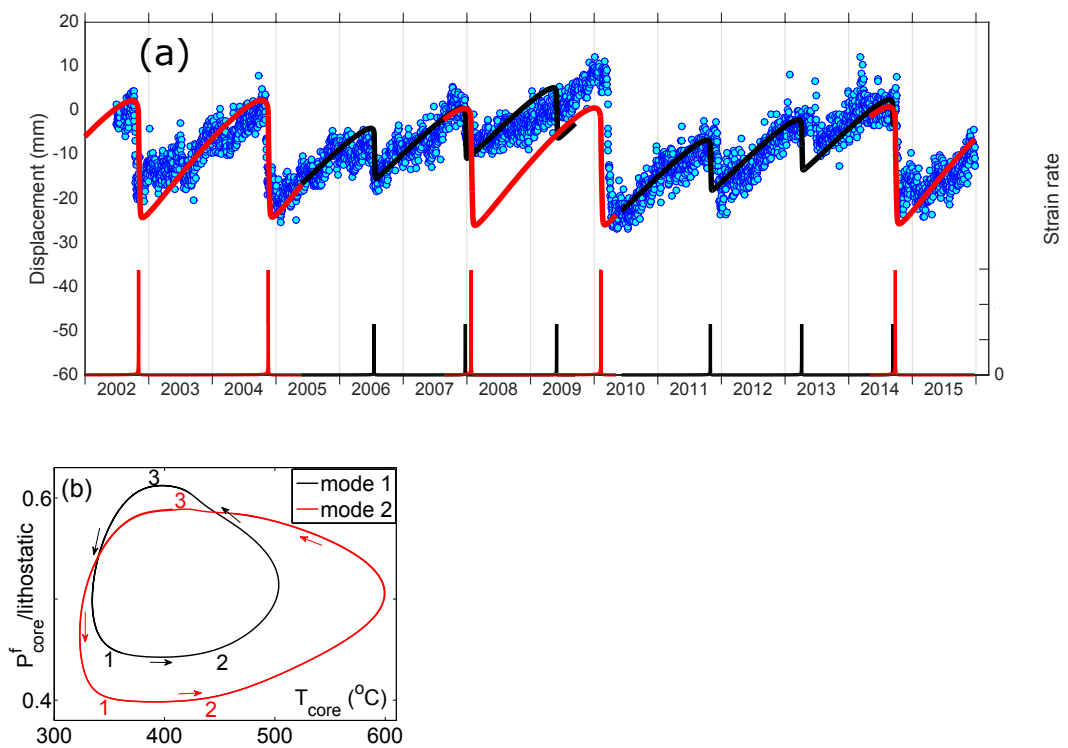

Figure 15. Irregular ETS sequence of Gisborne, New Zealand, station GISB [NAS ]. (a) The GPS data (blue dots) represent raw displacement data with its linear trend removed. The signal is non-periodic and the suggested fit consists of 2 modes. The first mode (red line) has higher displacement and strain rates (shown at the bottom) per event and a period of 2 years, and the second one (black line) has a period of about 14 months. Figure until end of 2013 is as published in June 2014 [POU 14b]. (b) Limit cycles of the two modes identified. Note that this bi-modal chaotic oscillator is driven from the elastic surrounding, with the transition from mode 1 to mode 2 being the natural mode of the elastic loading. The reverse transition requires additional excitation that would relax the applied shear stress, e.g. from nearby seismic events

in the vicinity of the system. Such an event was recorded at the end of 2007, causing the perturbation recorded by the GPS station, and shifting the sequence back to mode 1 of the oscillator.

as a vessel for the ultralocalized instability. In the case of brittle failure negligible additional energy is required as the system behaves in a near temperature-independent, close to criticality regime. In the case of ductile failure the shear zone is not necessarily at criticality through time but the fluid-like creep motions raise the local dissipation to eventually trigger an energy transformation in an ultralocalized PSZ. Under the assumption of microstructural steady state of the shear zone the latter instability is periodic. 
We have presented in Figure 13 a direct comparison of brittle and ductile/creep fractures in shear zones. In the brittle case it is known from field and laboratory observations that the PSZ represents an ultralocalised process zone, consisting of ultrafine particles that have usually undergone mechano-chemical degradation (gelification, decarbonation and dehydration reactions, melting, as thoroughly discussed in [TOR 11]). Therefore, although the mechanism of the fault zone formation is the material bifurcation from homogenous deformation [MUH 87], the formation of PSZ's should be considered the effect of the chemical-mechanical deformation following the onset of the initial structure.

The ductile shear zone in Figure 13 features a chemical transition from host rock to hanging rock and a strong grain size reduction towards the PSZ [HER 08]. The mechanism of localisation, although different from the brittle case, has the same style of energetic transition where microstructural changes are captured by their latent heat effects inside the PSZ. The main difference in terms of energetics is the time-scale of deformation, which we have shown to be governed by the energy equation and lastly the thermal activation.

In the brittle zone thermal activation is not considered important. However, a similar energetic transition may be taking place. The activation process can equally be triggered by a lowering of the critical threshold to near ambient condition through the access of fluids interacting with the host rock. It can be argued that in the brittle case the thermal time scale control is replaced by the mechanism of fluid transfer during fault slip. These mechanisms can be low temperature pressurisation effects [SIB 73, LAC 80, WIB 05, SUL 05, RIC 06] or high temperature chemical effects such as dehydration of minerals or decomposition of carbonates, theoretically studied recently [SUL 09, VEV 10, BRA 10, BRA 11b] and reported to take place in real faults [HIR 07, FAM 08] and experiments at laboratory conditions [HAN 07, FER 10, BRA 11a, PAO 11, COL 13]. Additional mechanisms that include dry phase transitions such as breakage can be formulated in a similar thermodynamic framework [EIN 07a, EIN 07b, LYA 14b, LYA 14a]. Therefore the above described framework illustrates an energy based formulation for instabilities of solid matter with emergent length scales of localisation.

This fundamental outcome is illustrated in the present approach by two scales of localisation naturally emerging and corresponding to two different energy regimes. These are the traditional Solid Mechanical localization during failure leading to a finite width shear band that can be described by internal variables and the Fluid Dynamical post-failure localization related to a phase change that counterintuitively leads to ultra thin shear zones within the master Solid Mechanical containment. We identify these fundamental outcomes to be a plausible explanation for the two scale localization phenomena described in this work. 


\section{.1. Poro-chemical model}

At high temperatures the solid $A B$ breaks down, producing excess $B$ fluid, and increasing the fluid pore pressure through a general fluid-release reaction of the form:

$$
\nu_{1} A B_{s} \rightleftharpoons \nu_{2} A_{s}+\nu_{3} B_{f}
$$

We assume the following relations for the partial molar reaction rates for the species,

$$
\begin{array}{r}
r_{A B}=-\left[\frac{\rho_{A B}}{M_{A B}}(1-\phi)(1-s)\right]^{\nu_{1}} k_{F} \exp \left(-Q_{F} / R T\right) \\
r_{A}=\left[\frac{\rho_{A}}{M_{A}}(1-\phi) s\right]^{\nu_{2}} k_{R} \exp \left(-Q_{R} / R T\right) \\
r_{B}=\left[\Delta \phi_{\text {chem }} \frac{\rho_{B}}{M_{B}}\right]^{\nu_{3}} k_{R} \exp \left(-Q_{R} / R T\right)
\end{array}
$$

From the stoichiometry of the considered reaction, Eq.(33), it should hold that:

$$
-\frac{r_{A B}}{\nu_{1}}=\frac{r_{A}}{\nu_{2}}=\frac{r_{B}}{\nu_{3}} .
$$

From Eqs. (34-35), and for $\nu_{1}=\nu_{2}=\nu_{3}=1$ we derive the poro-chemical model

$$
\begin{array}{r}
\Delta \phi_{\text {chem }}=A_{\phi} \frac{1-\phi_{0}}{1+\frac{\rho_{B}}{\rho_{A}} \frac{M_{A}}{M_{B}} \frac{1}{s}}, \\
s=\frac{\omega_{r e l}}{1+\omega_{r e l}}, \text { and } \\
r_{r e l}=\frac{\rho_{A B}}{\rho_{A}} \frac{M_{A}}{M_{A B}} K_{c} \exp \left(\frac{\Delta h}{R T}\right) .
\end{array}
$$

In Eqs. (36), $K_{c}=k_{F} / k_{R}$ is the ratio of the pre-exponential factors of the Arrhenius reaction rates and $\Delta h=Q_{R}-Q_{F}$ the difference of the forward and reverse activation energies. The parameter $A_{\phi}$ is a coefficient that determines the amount of the interconnected pore-volume (porosity) created due to the reaction. We assume that all the fluid generated contributes to the interconnected pore volume, and thus set $A_{\phi}=1$. 
Following these considerations, the rates of the forward $\left(\omega_{F}\right)$ and reverse $\left(\omega_{R}\right)$ first order reactions can be calculated to be

$$
\begin{array}{r}
r_{F}=r_{A B}=\frac{\rho_{A B}}{M_{A B}}(1-\phi)(1-s) k_{F} e^{-Q_{F} / R T} \\
r_{R}=r_{A} r_{B}=\frac{\rho_{A} \rho_{B}}{M_{A} M_{B}}(1-\phi) s \Delta \phi_{c h e m} k_{R} e^{-Q_{R} / R T} .
\end{array}
$$

Note that, for simplicity we have assumed in Eqs. (34) that the two products are produced with the same pre-exponential factor and activation energies. If this is not the case the above model should be modified accordingly. The net reaction rate would then be $r=r_{F}-r_{R} \frac{M_{A B}}{\rho_{A B}}$ (the reverse reaction rate was normalized with the reference concentration $\frac{\rho_{A B}}{M_{A B}}$ for dimensional purposes), which however would be essentially irreversible $\left(r_{F} \gg r_{R}\right)$ in the case $K_{c}=k_{F} / k_{R} \gg 1$.

\section{.2. Bibliographie}

[AlE 14] Alevizos S., Poulet T., Veveakis E., «Thermo-poro-mechanics of chemically active creeping faults. 1: Theory and steady state considerations », Journal of Geophysical Research: Solid Earth, vol. 119, n6, p. 4558-4582, 2014.

[AlL 12] Allam A. A., Ben-ZION Y., «Seismic velocity structures in the Southern California plate-boundary environment from double-difference tomography », Geophys. J. Int., , nº190, p. 1181-1196, 2012.

[BEN 03] Ben-Zion Y., Sammis C. G., "Characterization of Fault Zones», Pure Appl. Geophys., , n¹60, p. 677-715, 2003.

[BLA 08] Blasio F. D., Elverhøi A., «A model for frictional melt production beneath large rock avalanches. », J. Geophys. Res., vol. 113, pageF02014, 2008.

[BRA 10] Brantut N., Schubnel A., Corvisier J., Sarout J., «Thermochemical pressurization of faults during coseismic slip. », J. Geophys Res., vol. 115, pageB05314, 2010.

[BRA 11a] Brantut N., Han R., Shimamoto T., Findling N., Schubnel A., «Fast slip with inhibited temperature rise due to mineral dehydration: Evidence from experiments on gypsum. », Geology, vol. 39, n ${ }^{\circ}$, p. 59-62, 2011.

[BRA 11b] BRAntut N., Sulem J., SChubnel J., «Effect of dehydration reactions on earthquake nucleation: Stable sliding, slow transients and unstable slip. », J. Geophys Res., vol. 116, pageB05304, 2011.

[CEC 11] Cecinato F., Zervos A., Veveakis E., «A thermo-mechanical model for the catastrophic collapse of large landslides », International Journal for Numerical and Analytical Methods in Geomechanics, vol. 35, n 14, p. 1507-1535, John Wiley and Sons, Ltd., 2011. 


\section{non-peer reviewed EarthArXiv preprint}

[CHE 93] Chester F. M., Evans J. P., Biegel R. L., «Internal structure and weakening mechanisms of the San Andreas Fault», Journal of Geophysical Research: Solid Earth, vol. $98, n^{\circ}$ B1, p. $771-786,1993$.

[CHE 98] Chester F., Chester J., «Ultracataclasite structure and friction processes of the Punchbowl fault, San Andreas system, California. », Tectonophysics, p. 199-221, 1998.

[CHR 92] Chrysochoos A., Belmahjoub F., « Thermographic analysis of thermomechanical couplings », Archives Mechanics, vol. 44, n 1, p. 55-68, 1992.

[COL 13] Colletini C., Viti C., Tesei T., Mollo. S., « Thermal decomposition along natural carbonate faults duing earthquakes », Geology, pagedoi:10.1130/G34421.1, 2013.

[COR 04] Cornet F., Doan M., Moretti I., Borm G., « Drilling through the active Aigion fault: the aig10 well observatory ", Comptes Rendus Geosciences, vol. 336, n ${ }^{\circ} 4$ 5, p. 395-406, 2004.

[EIN 07a] EINAV I., «Breakage mechanics-Part I: Theory », Journal of the Mechanics and Physics of Solids, vol. 55, n 6, p. 1274 - 1297, 2007.

[EIN 07b] EINAV I., «Breakage mechanics-Part II: Modelling granular materials », Journal of the Mechanics and Physics of Solids, vol. 55, nº, p. 1298 - 1320, 2007.

[FAM 08] Famin V., Nakashima S., Boullier A.-M., Fujimoto K., Hirono T., «Earthquakes produce carbon dioxide in crustal faults », Earth. Plan. Sci. Let., vol. 265, p. 487497, 2008

[FAU 03] FAulkner D. R., Lewis A. C., RUtTER E. H., «On the internal structure and mechanics of large strike-slip fault zones: field observations of the Carboneras fault in southeastern Spain», Tectonophysics, , n³67, p. 235-251, 2003.

[FER 10] Ferri F., DiToro G., Hirose T., Shimamoto T., « Evidence of thermal pressurization in high-velocity friction experiments on smectite-rich gouges. », Terra Nova, vol. 22, n5, p. 347-353, 2010.

[FOW 97] Fowler A., Ed., Mathematical Models in the Applied Sciences, Cambridge University Press, 2 édition, 1997.

[FUJ 69] Fujita C., « On the non-linear equations $D u+e^{u}=0$ and $v_{t}=D v+e^{v}$.», Bull. Am. Math. Soc., vol. 75,, p. 132 - 135,, 1969.

[GOR 10] Goren L., Aharonov E., Anders M. H., «The long runout of the Heart Mountain landslide: Heating, pressurization, and carbonate decomposition », Journal of Geophysical Research: Solid Earth, vol. 115, nB10, p. n/a-n/a, 2010.

[Han 07] Han R., Shimamoto T., Hirose T., Ree J., Ando J., «Ultralow friction of carbonate faults caused by thermal decomposition. », Science, vol. 316, p. 878-881, 2007.

[Her 08] Herwegh M., Hurzeler J., Pfiffner O., Schmid S., Abart R., Ebert A., «The Glarus Thrust: excursion guide and report of a field trip of the swiss tectonic studies groups », Swiss Journal of Geosciences, vol. 101, n², p. 323-340, 2008.

[HIL 62] HILl R., «Acceleration waves in solids. », J. Mech. Phys. Solids, vol. 10, p. 1-16, 1962. 
[HIR 07] HiRono T., ET AL., «A chemical kinetic approach to estimate dynamic shear stress during the 1999 Taiwan Chi-Chi earthquake. », Geophys. Res. Lett., vol. 34, pageL19308, 2007.

[HOL 11] Holdsworth R., van Diggelen E., Spiers C., De Bresser J., Walker R., BOWEN L., «Fault rocks from the \{SAFOD $\}$ core samples: Implications for weakening at shallow depths along the San Andreas Fault, California », Journal of Structural Geology, vol. 33, n², p. 132 - 144, 2011.

[KEN 97] KenNedy L., Logan J., «The role of veining and dissolution in the evolution of fine -grained ylonites: the McConnell Thrust, Alberta », J. Struct. Geology, vol. 19, n 6 , p. 785-797, 1997.

[L. 07] L. G., Aharonov E., «Long runout landslides: The role of frictional heating and hydraulic diffusivity. », Geophys. Res. Lett., vol. 34, pageL07301, 2007.

[LAC 80] LACHENBRUCH A., «Frictional heating, fluid pressure and the resistance to fault motion. », J. Geophys. Res., vol. 85, p. 6097-6112, 1980.

[LYA 14a] LYAKHOVSKY V., BEN-Zion Y., «A continuum damage-breakage faulting model accounting for solid-granular transitions », Pure Appl. Geophys., pageSubmitted, 2014.

[LYA 14b] LYAKhOVsky V., BeN-Zion Y., « Damage-Breakage rheology model and solidgranular transition near brittle instability », J. Mech. Phys. Solids, , n64, p. 184-197, 2014.

[MAN 66] MANDEl J., «Conditions de stabilite et postulate de Drucker.», Rheology and Soil Mechanics, p. 58-67, 1966.

[MUH 87] Muhlhaus H., VARdoulakis I., «Thickness of shear bands in granular materials. », Geotechnique, vol. 37, n³, p. 271-283, 1987.

[NAS ] NASA, « jet propulsion laboratory, CalTech: http://sideshow.jpl.nasa.gov/post/series.html ».

[NOD 05] Noda H., Shimamoto T., « Thermal pressurization and slip-weakening distance of a fault: An example of the Hanaore fault, Southwest Japan», Bull. Seism. Soc. Am., vol. $95, \mathrm{n}^{\circ} 4,2005$.

[PaO 11] Paola N. D., Hirose T., Mitchell T., Toro G. D., Togo T., Shimamoto T., «Fault lubrication and earthquake propagation in thermally unstable rocks. », Geology, vol. $39, n^{\circ} 1$, p. 35-38, 2011.

[PAP 11] PaPAnicolopulos S., VeVeakis E., « Sliding and rolling dissipation in Cosserat plasticity », Granular Matter, vol. 13, n³, p. 197-204, 2011.

[PER 66] PERZYNA P., «Fundamental problems in viscoplasticity. », Adv. Appl. Mech., vol. 9, p. 243-377, 1966

[POU 14a] Poulet T., Veveakis E., Regenauer-Lieb K., Yuen D. A., «Thermo-poromechanics of chemically active creeping faults: 3 . The role of serpentinite in episodic tremor and slip sequences, and transition to chaos », Journal of Geophysical Research: Solid Earth, vol. 119, nº ${ }^{\circ}$, p. 4606-4625, 2014.

[POU 14b] Poulet T., Veveakis M., Herwegh M., Buckingham T., RegenauerLIEB K., «Modeling episodic fluid-release events in the ductile carbonates of the Glarus thrust », Geophysical Research Letters, vol. 41, n²0, p. 7121-7128, 2014. 
[RAT 17a] Rattez H., Stefanou I., SUlem J., «Thermo-Hydro-Mechanical couplings and strain localisation in 3D continua with microstructure . Part I: Theory and linear stability analysis », submitted to Journal of Mechanics and Physics of Solids, 2017.

[RAT 17b] Rattez H., Stefanou I., Sulem J., Veveakis M., Poulet T., «ThermoHydro-Mechanical couplings and strain localisation in 3D continua with microstructure . Part II: Numerical implementation and post-bifurcation analysis », submitted to Journal of Mechanics and Physics of Solids, 2017.

[RAT 17c] Rattez H., Stefanou I., Veveakis M., Poulet T., Sulem J., « Numerical analysis of strain localization in Rocks with THM couplings: Benchmark tests and microstructure », Submitted to Rock Mechanics and Rock Engineering, 2017.

[REG 09] Regenauer-Lieb K., Yuen D., Fusseis F., «Landslides, Ice Quakes, Earthquakes: A Thermodynamic Approach to Surface Instabilities », Pure. Appl. Geophys, vol. 166, n 10-11, p. 1885-1908, 2009.

[REG 13a] Regenauer-Lieb K., Veveakis M., Poulet T., Wellmann F., Karrech A., Liu J., Hauser J., Schrank C., Gaede O., Trefry M., «Multiscale coupling and multiphysics approaches in earth sciences: Applications », Journal of Coupled Systems and Multiscale Dynamics, vol. 1, n³, pagedoi:10.1166/jcsmd.2013.1021, 2013.

[REG 13b] Regenauer-Lieb K., Veveakis M., Poulet T., Wellmann F., Karrech A., Liu J., Hauser J., Schrank C., Gaede O., Trefry M., « Multiscale coupling and multiphysics approaches in earth sciences: Theory », Journal of Coupled Systems and Multiscale Dynamics, , vol. 1, ${ }^{\circ} 1$, p. 49-73, 2013.

[REI 64] ReIner M., «The Deborah Number», Physics Today, p. 152-153, 1964.

[RIC 06] RICE J. R., «Heating and weakening of faults during earthquake slip », J. Geophys. Res., vol. 111, pageB05311, 2006

[ROS 00] Rosakis P., Rosakis A., Ravichandran G., Hodowany J., «A thermodynamic internal variable model for the partition of plastic work into heat and stored energy in metals. », J. Mech. Phys. Solids, vol. 48, p. 581-607, 2000.

[ROW 12] Rowe C., Fagereng A., Miller J., Mapani B., « Signature of coseismic decarbonation in dolomitic fault rocks of the Naukluft Thrust, Namibia », Earth Plan. Sci. Let., vol. 333-334, p. 200-210, 2012.

[RUD 75] RUDNICKI JW R. J., «Conditions for the localization of deformation in pressure sensitive dilatant materials. », J. Mech. Phys. Solids, vol. 23, p. 371-394, 1975.

[SIB 73] SIBSON R., «Interaction between temperature and pore-fluid pressure during earthquake faulting - a mechanism for partial or total stress relief », Nature, vol. 243, p. 66-68, 1973.

[SIB 03] Sibson R., «Thickness of the Seismic Slip Zone», Bull. Seism. Soc. Am., vol. 93, p. $1169-1178,2003$.

[STE 14] Stefanou I., Sulem J., "Chemically induced compaction bands: Triggering conditions and band thickness », Journal of Geophysical Research: Solid Earth, vol. 119, n², p. 880-899, 2014. 
[SUl 05] Sulem J. Vardoulakis I. O. H., Perdikatsis V., « Thermo-poro-mechanical Properties of the Aigion Fault Clayey Gouge - Application to the Analysis of Shear Heating and Fluid Pressurization », Soils and Foundations, vol. 45, p. 97-108, 2005.

[SUL 07] Sulem J., LAZAR P., VARdoulaKis I., «Thermo-Poro-Mechanical Properties of Clayey Gouge and Application to Rapid Fault Shearing. », Int. J. Num. Anal. Meth. Geomechanics, vol. 31, n³, p. 523-540., 2007.

[SUL 09] SUlEM J., FAMIN V., « Thermal decomposition of carbonates in fault zones: slipweakening and temperature-limiting effects. », J. Geophys. Res., vol. 114, pageB03309, 2009.

[SUL 11] Sulem J., Stefanou I., Veveakis E., «Stability analysis of undrained adiabatic shearing of a rock layer with Cosserat microstructure. », Granular Matter, vol. 13, n 3 , p. 261-268, 2011.

[TAY 34] TAYLOR G., QUINNEY H., « The latent energy remaining in a metal after cold working », Proc. R. Soc., Ser. A., vol. 143, p. 307 - 326., 1934.

[TOB 45] Tовоlsky A., Andrews R., « Systems manifesting superposed elastic and viscous behaviour», J.Chem. Phys., vol. 13, n 1, p. 3-27, 1945.

[TOR 11] Toro G. D., Han R., Hirose T., DePaola N., Nielsen S., Mizoguchi K., Ferri F., Cocco M., Shimamoto T., «Fault lubrication during earthquakes », Nature, vol. 471, p. 494-498, 2011.

[TOW 09] Townend J., « Drilling, Sampling, and Monitoring the Alpine Fault: Deep Fault Drilling Project-Alpine Fault, New Zealand; Franz Josef, New Zealand, 22-28 March 2009 », Eos, Transactions American Geophysical Union, vol. 90, n³6, p. 312-312, 2009.

[VAR 2a] VARDOUlaKIS I., "Dynamic thermo-poro-mechanical analysis of catastrophic landslides. », Geotechnique, vol. 52, p. 157-171, 2002a.

[VAR 95] VARDOUla IS I., SUlEM J., Eds., Bifurcation Analysis in Geomechanics, Blankie Acc. and Professional, 1995.

[VEV 07] VeVeakis E., VARdoulakis I., DiToro. G., «Thermoporomechanics of creeping landslides: The 1963 Vaiont slide, northern Italy. », J. Geophys. Res., vol. 112, pageF03026, 2007.

[VEV 10] Veveakis E., Alevizos S., Vardoulakis. I., «Chemical reaction capping of thermal instabilities during shear of frictional faults. », J. Mech. Phys. Solids, vol. 58, p. $1175-1194,2010$.

[VEV 12] Veveakis E., Sulem J., Stefanou I., «Modeling of fault gouges with Cosserat Continuum Mechanics: Influence of thermal pressurization and chemical decomposition as coseismic weakening mechanisms », J. Struct. Geology, vol. 38, p. 254-264, 2012.

[VEV 13] VeVEAKIS E., STEFANOU I., SUlEM J., «Failure in shear bands for granular materials: thermo-hydro-chemo-mechanical effects », Geotechnique Let., vol. 3, n² ${ }^{\circ}$, p. 31-36, 2013.

[VEV 14a] Veveakis E., Poulet T., Alevizos S., «Thermo-poro-mechanics of chemically active creeping faults: 2 . Transient considerations », Journal of Geophysical Research: Solid Earth, vol. 119, nº, p. 4583-4605, 2014. 
[VEV 14b] VeVeakis E., Regenauer-Lieb K., «The fluid dynamics of solid mechanical shear zones », Pure Appl. Geophys., vol. 171, p. 3159-3174, 2014.

[VEV 14c] Veveakis E., Regenauer-Lieb K., Weinberg R., « Ductile compaction of partially molten rocks: the effect of non-linear viscous rheology on instability and segregation », Geophysical Journal International, vol. 200, n¹, p. 519-523, 2014.

[VEV 15] Veveakis E., Regenauer-Lieb, «Cnoidal Waves in Solids », Journal of Mechanics and Physics of Solids, vol. 78, p. 231-248, 2015.

[WIB 03] Wibberley C. A. J., Shimamoto T., «Internal structure and permeability of major strike-slip fault zones: The median tectonic line in mid prefecture, southwest Japan », J. Struct. Geol., vol. 25, p. 59-78, 2003.

[WIB 05] Wibberley C., Shimamoto T., «Earthquake slip weakening and asperities explained by thermal pressurization. », Nature, vol. 426, n 4 , p. 689-692, 2005.

[YUE 77] YUEN D., SChUbeRt G., «Asthenospheric shear flow: thermally stable or unstable?», Geophys. Res. Lett, vol. 4, n¹1, p. 503-506, 1977. 